\title{
On convergent sequences in dual groups
}

\author{
M.V. Ferrer ${ }^{*} \quad$ S. Hernández ${ }^{\dagger} \quad$ M. Tkachenko ${ }^{\ddagger}$
}

December 20, 2019

\begin{abstract}
We provide some characterizations of precompact abelian groups $G$ whose dual group $G_{p}^{\wedge}$ endowed with the pointwise convergence topology on elements of $G$ contains a nontrivial convergent sequence. In the special case of precompact abelian torsion groups $G$, we characterize the existence of a nontrivial convergent sequence in $G_{p}^{\wedge}$ by the following property of $G$ : No infinite Hausdorff quotient group of $G$ is countable. Also, we present an example of a dense subgroup $G$ of the compact metrizable group $\mathbb{Z}(2)^{\omega}$ such that $G$ is of the first category in itself, has measure zero, but the dual group $G_{p}^{\wedge}$ does not contain infinite compact subsets. This complements a result of J.E. Hart and K. Kunen (2005) on convergent sequences in dual groups. Making use of the group $G$ we construct the first example of a precompact Pontryagin reflexive abelian group which is of the first Baire category.
\end{abstract}

Keywords: Reflexive; Precompact; Pseudocompact; Baire property; Convergent sequence

MSC: Primary 43A40, 22D35; Secondary 22C05, 54E52, 54C10

*This author was partially supported by the Generalitat Valenciana, grant $\mathrm{GV} / 2018 / 110$

${ }^{\dagger}$ This author was partially supported by the Spanish Ministerio de Economía y Competitividad, grant MTM2016-77143-P (AEI/FEDER, EU)

† The article was finished during the visit of the third listed author to the Universitat Jaume I, Spain, in June, 2019. He expresses his gratitude to the hosts for financial support and kind attention. 


\section{Introduction}

A topological group $G$ is said precompact (resp., pseudocompact) if it is topologically isomorphic to a dense subgroup of a compact topological group (resp., every continuous real-valued function on $G$ is bounded). Here, we study the class of precompact topological abelian groups $G$ such that the dual group $G_{p}^{\wedge}$ endowed with the pointwise convergence topology on elements of $G$ contains a nontrivial convergent sequence. It is well known that a group $G$ from this class cannot be compact (see [15, Theorem 1.2]). A more general result follows from [18, Proposition 4.4]: If $G_{p}^{\wedge}$ contains infinite compact subsets then $G$ is not pseudocompact. An even more general fact is established in [12]: If $G$ has the Baire property, then $G_{p}^{\wedge}$ does not contain nontrivial convergent sequences. We conjecture that $G_{p}^{\wedge}$ does not contain infinite compact subsets in this case (see Problem 3.10). This conjecture has been proved in [4, Theorem 3.3] for bounded torsion groups $G$.

Therefore, we have to consider only precompact groups of the first Baire category, i.e. the groups which can be covered by countably many nowhere dense subsets. In Theorem 2.3 we show that the compact metrizable abelian group $\mathbb{Z}(2)^{\omega}$ contains a dense subgroup $G$ such that $G$ is of the first category in itself, but every convergent sequence in the dual group $G_{p}^{\wedge}$ is trivial. Furthermore, since $G_{p}^{\wedge}$ is countable, all compact subsets of $G_{p}^{\wedge}$ are finite. This complements [17, Theorem 1.6] by J.E. Hart and K. Kunen. We conclude that the property of a precompact abelian group $G$ to be 'small' (to be Haar nullset in the completion of $G$ or to be of the first category in itself, or both) does not guarantee the existence of nontrivial convergent sequences in $G_{p}^{\wedge}$.

In the special case when $G$ is an infinite topological subgroup of the circle group $\mathbb{T}$, our study is intimately related to the so-called characterized subgroups of $\mathbb{T}$ (see the articles $[7,8]$ and the references therein). In this case, the dual group $G^{\wedge}$ is algebraically isomorphic to the group $\mathbb{Z}$ of integers. Given a strictly increasing sequence $B=\left(n_{k}\right)_{k \in \omega}$ of positive integers, one defines $C_{B}^{\mathbb{T}}$ to be the set of all $x \in \mathbb{T}$ such that $x^{n_{k}} \rightarrow 1$ when $k \rightarrow \infty$. A subgroup $G$ of $\mathbb{T}$ is called characterized if $G=C_{B}^{\mathbb{T}}$, for some sequence $B \subset \omega$. It is clear from the definition that the sequence $B$ converges to the identity of the dual group $\mathbb{Z}=\left(C_{B}^{\mathbb{T}}\right)_{p}^{\wedge}$ and that $C_{B}^{\mathbb{T}}$ is the biggest subgroup of $\mathbb{T}$ for which

$B$ converges. It is known that $C_{B}^{\mathbb{T}}$ has measure zero in $\mathbb{T}$, for each $B \subset \omega$ [6, Lemma 3.10]. This is one of very few general results about characterized subgroups of $\mathbb{T}$, though the articles dedicated to their study abound. It is also worth mentioning that the index $[\mathbb{T}: G]$ of a characterized subgroup 
$G \leq \mathbb{T}$ is uncountable - otherwise the circle group could be covered by a countable family of nullsets, which is impossible. Needless to say, no internal description of characterized subgroups of $\mathbb{T}$ is available now.

Summing up, searching for nontrivial convergent sequences in the dual groups of dense subgroups of compact connected groups presents considerable difficulties. Here, we tackle this question and present some conditions guaranteeing that the dual group $G_{p}^{\wedge}$ of a precompact abelian group $G$ contains a nontrivial convergent sequence. Clearly, if $G$ is a countable infinite precompact abelian group, then the dual group $G_{p}^{\wedge}$ has a countable base and is not discrete. Hence $G_{p}^{\wedge}$ contains nontrivial convergent sequences. We use this simple observation in the proofs of several results here. We focus our attention on the study of the duals of precompact abelian torsion groups $G$. In this special case, we characterize in Theorem 2.7 the existence of nontrivial convergent sequences in $G_{p}^{\wedge}$ by the following property of $G$ : No infinite quotient group of $G$ is countable. Finally, an example of a reflexive, precompact, abelian group of the first Baire category is provided.

\subsection{Notation and terminology}

The identity element of a group $G$ is denoted by $e_{G}$ or simply $e$ if no confusion is possible. A character of a group $G$ is an arbitrary homomorphism of $G$ to the circle group $\mathbb{T}$. The latter group is identified with the multiplicative subgroup of the complex numbers $z$ with $|z|=1$. If $G$ is a topological group, a continuous character of $G$ is a continuous homomorphism of $G$ to $\mathbb{T}$ provided the latter group carries its usual compact topology inherited from the complex plane $\mathbb{C}$. We also set $V_{1}=\{z \in \mathbb{T}:-\pi / 2<\operatorname{Arg}(z)<\pi / 2\}$.

For a given topological group $G$, its dual group is denoted by $G^{\wedge}$ (see [22]). The dual group consists of the continuous characters of $G$ with the pointwise multiplication, $\left(\chi_{1} \cdot \chi_{2}\right)(x)=\chi_{1}(x) \cdot \chi_{2}(x)$ for each $x \in G$. The identity element of $G^{\wedge}$ is the constant homomorphism $e: G \rightarrow \mathbb{T}, e(x)=1$ for each $x \in G$. Here 1 is the identity element of $\mathbb{T}$.

In this article, the dual group $G^{\wedge}$ will always be endowed with the pointwise convergence topology whose local base at the identity $e$ is formed by the sets

$$
O\left(x_{1}, \ldots, x_{n}\right)=\left\{\chi \in G^{\wedge}: \chi\left(x_{k}\right) \in V_{1} \text { for each } k=1, \ldots, n\right\},
$$

where $n$ is a positive integer and $x_{1}, \ldots, x_{n} \in G$. The group $G^{\wedge}$ equipped 
with the pointwise convergence topology on $G$ is denoted by $\left(G^{\wedge}, t_{p}(G)\right)$ or by $G_{p}^{\wedge}$, for short, when there is no possible confusion.

There exist infinite Hausdorff topological abelian groups $G$ with the trivial dual group $G^{\wedge}$ (see [23]). If, however, the group $G$ is precompact (equivalently, is topologically isomorphic to a dense subgroup of a compact topological group), then the continuous characters of $G$ separate points of $G$. Furthermore, in this case, the topology of $G$ coincides with the topology of pointwise convergence on elements of the dual group $G^{\wedge}$. This follows from a theorem of Comfort and Ross (see [5, Theorem 1.2]).

If $N$ is a subgroup of a topological group $G$, we denote by $N^{\perp}$ the annihilator of $N$, i.e. the subgroup of $G^{\wedge}$ which consists of all characters $\chi \in G^{\wedge}$ satisfying $\chi(N)=\{1\}$. The group $N^{\perp}$ is always closed in $G_{p}^{\wedge}$. Similarly, if $M$ is a subgroup of $G^{\wedge}, M^{\perp}$ denotes the annihilator of $M$ consisting of all elements $x \in G$ satisfying $\chi(x)=1$ for each $\chi \in M$. It is clear that $M^{\perp}$ is the intersection of the kernels of the characters of $M$. Again, $M^{\perp}$ is a closed subgroup of $G$. The notion of annihilator will be only used in Proposition 2.5, where we present a characterization of precompact abelian groups which have countably infinite Hausdorff quotients.

Let $f: G \rightarrow H$ be a continuous homomorphism of topological groups. We define the dual homomorphism $f^{\wedge}: H_{p}^{\wedge} \rightarrow G_{p}^{\wedge}$ by letting $f^{\wedge}(\chi)=\chi \circ f$ for each $\chi \in H_{p}^{\wedge}$. It is easy to see that $f^{\wedge}$ is continuous. It is also known that $f^{\wedge}$ is injective if $f(G)=H$ and that $f^{\wedge}$ is onto if $f$ is a topological monomorphism and $H$ (hence, $G$ ) is precompact and abelian [9, 22].

\section{Duals of precompact torsion groups}

The following dichotomy for homogeneous spaces is a kind of the topological folklore (see [21, Theorem 2.3]).

Lemma 2.1 If a homogeneous space $X$ is not Baire, then $X$ is of the first category in itself.

In what follows we identify the two-element cyclic group $\mathbb{Z}(2)$ with the subgroup $\{1,-1\}$ of the multiplicative circle group $\mathbb{T}$. We start with a simple and well known lemma that will be applied in the proof of Theorem 2.3. We include a short proof of it here for completeness sake.

Lemma 2.2 Let $\chi: \mathbb{Z}(2)^{A} \rightarrow \mathbb{T}$ be a homomorphism, where $|A|<\omega$. Then there exists a set $B \subset A$ such that $\chi(x)=\prod_{i \in B} x(i)$, for each $x \in \mathbb{Z}(2)^{A}$. 
Proof. If $H=\prod_{i \in A} H_{i}$ is a product of finitely many Abelian groups and $\chi: H \rightarrow \mathbb{T}$ is a homomorphism, then there exist homomorphisms $\chi_{i}: H_{i} \rightarrow$ $\mathbb{T}$, for $i \in A$, such that $\chi(x)=\prod_{i \in A} \chi_{i}\left(x_{i}\right)$ for each $x \in \mathbb{Z}(2)^{A}$. Since every homomorphism of $\mathbb{Z}(2)$ to $\mathbb{T}$ is either trivial or a monomorphism, the conclusion of the lemma is now immediate.

A subset $B$ of a Tychonoff space $X$ is called bounded in $X$ if the image $f(B)$ is a bounded subset of the real line, for every continuous real-valued function $f$ on $X$ (see [2, Section 6.10]). It is clear that all compact subsets of $X$ are bounded. Conversely, in a Dieudonné complete space $X$ (that is, complete with respect to the finest uniformity compatible with the topology of $X)$, the closure of every bounded subset is compact [2, Proposition 6.10.1 c)].

In the following theorem, we denote by $\mu$ the Haar measure of the compact group $\mathbb{Z}(2)^{\omega}$ (see [16, Chapter 10]).

Theorem 2.3 There exists an infinite first category subgroup $G$ of the compact group $\mathbb{Z}(2)^{\omega}$ such that $\mu(G)=0$ and every bounded subset of the dual group $G_{p}^{\wedge}$ is finite. In particular, $G_{p}^{\wedge}$ does not contain non-trivial convergent sequences.

Proof. Let call a subset $A$ of $\omega$ thin if

$$
\lim _{k \rightarrow \infty} \frac{|k \cap A|}{k}=0
$$

where each positive integer $k \in \omega$ is identified with the set $\{0, \ldots, k-1\}$. For an element $x \in \mathbb{Z}(2)^{\omega}$, let

$$
\operatorname{supp}(x)=\{n \in \omega: x(n)=1\} .
$$

Denote by $G$ the set of all $x \in \mathbb{Z}(2)^{\omega}$ such that $\operatorname{supp}(x)$ is a thin subset of $\omega$. It is clear that $G$ is a dense subgroup of $\mathbb{Z}(2)^{\omega}$ and that $G$ is precompact.

First, we claim that $G$ is of the first category in $\mathbb{Z}(2)^{\omega}$. Indeed, for positive integers $m$ and $N$, let

$$
O_{m, N}=\left\{x \in \mathbb{Z}(2)^{\omega}: \exists k \geq m \text { such that } \frac{|\operatorname{supp}(x) \cap k|}{k} \geq 1 / N\right\} .
$$

It is easy to see that the sets $O_{m, N}$ are open and dense in $\mathbb{Z}(2)^{\omega}$ whenever $N \geq 2$, while our definition of $G$ implies that $G$ is disjoint from the set 
$\bigcup_{m, N \geq 2} O_{m, N}$. Hence $G$ is contained in the first category set $\bigcup_{m, N \geq 2} F_{m, N}$, where $F_{m, N}=\mathbb{Z}(2)^{\omega} \backslash O_{m, N}$. This proves our claim.

Let us show that $\mu(G)=0$, where $\mu$ is the Haar measure on the compact group $\mathbb{Z}(2)^{\omega}$. It is easy to see that $G$ is measurable. Indeed, every set $O_{m, N}$ is open in the compact group $\mathbb{Z}(2)^{\omega}$ and, hence, is measurable. Hence the sets $F_{m, N}=\mathbb{Z}(2)^{\omega} \backslash O_{m, N}$ are also measurable. The definition of the group $G$ implies that

$$
G=\bigcap_{N=1}^{\infty} \bigcup_{m=0}^{\infty} F_{m, N},
$$

whence it follows that $G$ is measurable. Since the index of $G$ in $\mathbb{Z}(2)^{\omega}$ is infinite, no finite number of cosets of $G$ in $\mathbb{Z}(2)^{\omega}$ covers the group $\mathbb{Z}(2)^{\omega}$. Hence, by the additivity of $\mu$, we conclude that $\mu(G)=0$.

Now we verify that the dual group $G_{p}^{\wedge}$ does not contain non-trivial sequences converging to the identity element of $G_{p}^{\wedge}$. Consider a sequence $\left\{\chi_{n}: n \in \omega\right\} \subseteq G_{p}^{\wedge}$. We can assume without loss of generality that $\chi_{n} \neq \chi_{m}$ if $n \neq m$. Since $G$ is dense in $\mathbb{Z}(2)^{\omega}$, every $\chi_{n}$ extends to a continuous character on $\mathbb{Z}(2)^{\omega}$. We denote this extension by $\varphi_{n}$, for each $n \in \omega$. According to $\left[20\right.$, Theorem $\left.\mathrm{D}_{1}\right]$, every $\varphi_{n}$ depends on finitely many coordinates, i.e. one can find a finite set $A_{n} \subset \omega$ and a character $\psi_{n}$ on $\mathbb{Z}(2)^{A_{n}}$ such that $\varphi_{n}=\psi_{n} \circ \pi_{A_{n}}$, where $\pi_{A_{n}}: \mathbb{Z}(2)^{\omega} \rightarrow \mathbb{Z}(2)^{A_{n}}$ is the projection. Clearly, we can assume that $A_{n}$ is a minimal (by inclusion) set with this property. It then follows from Lemma 2.2 that $\psi_{n}(x)=\prod_{i \in A_{n}} x(i)$, for each $x \in \mathbb{Z}_{2}^{A_{n}}$.

For every finite $A \subset \omega$, there exist only finitely many homomorphisms of $\mathbb{Z}(2)^{A}$ to $\mathbb{T}$. Hence, choosing a subsequence of $\left\{\chi_{n}: n \in \omega\right\}$, if necessary, we can assume that the sets $A_{n}$ are non-empty and that each $A_{n}$ is not covered by the sets $A_{m}$ with $m<n$. For every $n \in \omega$, let $k_{n}$ be the biggest element of the complement $A_{n} \backslash \bigcup_{m<n} A_{m}$. Choosing a subsequence of $\left\{\chi_{n}: n \in \omega\right\}$ once again, we can assume additionally that the set $K=\left\{k_{n}: n \in \omega\right\}$ is thin.

Let us define an element $x^{*} \in G$ as follows. Choose $x_{0} \in \mathbb{Z}(2)^{A_{0}}$ such that $\psi_{0}\left(x_{0}\right)=-1$ and $x_{0}(k)=1$ for each $k \in A_{0}$ distinct from $k_{0}$. Assume that for some $n \in \omega$, we have defined elements $x_{i} \in \mathbb{Z}(2)^{A_{i}}$ with $i \leq n$ satisfying the following conditions:

(a) $x_{i}(k)=x_{j}(k)$ whenever $j<i$ and $k \in A_{i} \cap A_{j}$;

(b) if $x_{i}(k)=-1$, then $k=k_{j}$ for some $j \leq i$; 
(c) $\psi_{i}\left(x_{i}\right)=-1$.

Let $y_{1}$ and $y_{2}$ be elements of $\mathbb{Z}(2)^{A_{n+1}}$ such that $y_{1}(k)=y_{2}(k)=x_{i}(k)$ whenever $k \in A_{n+1} \cap A_{i}$ for some $i \leq n, y_{1}(k)=y_{2}(k)=1$ for each $k \in A_{n+1} \backslash$ $\bigcup_{i \leq n} A_{i}$ distinct from $k_{n+1}$, and $y_{1}\left(k_{n+1}\right) \neq y_{2}\left(k_{n+1}\right)$. It follows from the choice of $A_{n+1}$ and $k_{n+1} \in A_{n+1}$ that $\psi_{n+1}\left(y_{1}\right) \neq \psi_{n+1}\left(y_{2}\right)$. Hence $\psi_{n+1}\left(y_{i}\right)=$ -1 for some $i \in\{1,2\}$. We put $x_{n+1}=y_{i}$. Then the elements $x_{0}, x_{1}, \ldots, x_{n+1}$ satisfy conditions (a)-(c).

Denote by $x^{*}$ an element of $\mathbb{Z}(2)^{\omega}$ such that $\pi_{A_{n}}\left(x^{*}\right)=x_{n}$ for each $n \in \omega$ (we apply (a) here) and $x^{*}(k)=1$ if $k \in \omega \backslash \bigcup_{n \in \omega} A_{n}$. Since the set $K=\left\{k_{n}\right.$ : $n \in \omega\}$ is thin, (b) implies that $\operatorname{supp}\left(x^{*}\right) \subseteq K$ and hence $x^{*} \in G$. It follows from (c) and our definition of $x^{*}$ that $\chi_{n}\left(x^{*}\right)=\psi_{n}\left(\pi_{A_{n}}\left(x^{*}\right)\right)=\psi_{n}\left(x_{n}\right)=-1$, for each $n \in \omega$. Therefore, the sequence $\left\{\chi_{n}: n \in \omega\right\}$ does not converge to the identity in $G_{p}^{\wedge}$. This implies that $G$ does not contain non-trivial convergent sequences at all.

Since $G$ is second countable and precompact, the dual group $G_{p}^{\wedge}$ is countable. Suppose that $B$ is an infinite bounded subset of $G_{p}^{\wedge}$. Then so is $\bar{B}$, the closure of $B$ in $G_{p}^{\wedge}$. Also, it follows from $\left|G_{p}^{\wedge}\right|=\omega$ that the space $G_{p}^{\wedge}$ is Lindelöf and, hence, Dieudonné complete. Therefore, $\bar{B}$ is compact. But every countably infinite compact space contains non-trivial convergent sequences, which is a contradiction.

Remark 2.4 We have shown in Theorem 2.3 that all bounded subsets of $G_{p}^{\wedge}$ are finite. This is equivalent to saying that every infinite set $X \subseteq G_{p}^{\wedge}$ contains an infinite subset $D$ such that $D$ is closed and discrete in $G_{p}^{\wedge}$ and $C$-embedded in $G_{p}^{\wedge}$. The equivalence follows immediately from the fact that the space $G_{p}^{\wedge}$ is countable and regular, hence, normal.

Proposition 2.5 Let $G$ be a precompact topological abelian group. The following conditions are equivalent:

(1) G has a countably infinite Hausdorff quotient;

(2) G has a countably infinite Hausdorff homomorphic image;

(3) $G_{p}^{\wedge}$ contains an infinite metrizable subgroup.

Proof. (2) $\Rightarrow(1)$. Let $\varphi: G \longrightarrow H$ be a continuous homomorphism onto a countably infinite Hausdorff topological group $H$. Then $N=\operatorname{ker} \varphi$ is a closed 
subgroup of $G$. Let $\pi: G \rightarrow G / N$ be the quotient homomorphism. Clearly, there is a continuous homomorphism $\varphi_{N}: G / N \longrightarrow H$ such that $\varphi=\varphi_{N} \circ \pi$. It is clear from the definition that $\varphi_{N}$ is one-to-one, which implies that $G / N$ is countable and Hausdorff.

$(1) \Rightarrow(3)$. Let $\pi: G \longrightarrow H$ be a quotient homomorphism onto a countably infinite Hausdorff topological group $H$. Clearly $H$ is precompact. Hence $H_{p}^{\wedge}$ is an infinite metrizable group and $\pi^{\wedge}: H_{p}^{\wedge} \rightarrow G_{p}^{\wedge}$ is a monomorphism, which is easily seen to be a topological embedding in $G_{p}^{\wedge}$. This shows that $\pi^{\wedge}\left(H_{p}^{\wedge}\right)$ is an infinite metrizable subgroup of $G_{p}^{\wedge}$.

$(3) \Rightarrow(2)$. Let $\Gamma$ be an infinite metrizable subgroup of $G_{p}^{\wedge}$. Then so is $\bar{\Gamma}$, the closure of $\Gamma$ in $G_{p}^{\wedge}$ (see [2, Proposition 1.4.16]). Hence we can assume that $\Gamma$ is closed in $G_{p}^{\wedge}$. Let $i: \Gamma \rightarrow G_{p}^{\wedge}$ be the identity embedding. Then the dual homomorphism $i^{\wedge}:\left(G_{p}^{\wedge}\right)_{p}^{\wedge} \rightarrow \Gamma_{p}^{\wedge}$ is continuous and surjective, while the kernel of $i^{\wedge}$ is $\Gamma^{\perp}$. According to [24], the canonical evaluation mapping of $G$ to $\left(G_{p}^{\wedge}\right)_{p}^{\wedge}$ is a topological isomorphism, so we can identify the groups $G$ and $\left(G_{p}^{\wedge}\right)_{p}^{\wedge}$. We have thus shown that the abstract groups $G / \Gamma^{\perp}$ and $\Gamma^{\wedge}$ are isomorphic. Since the latter group is countable and infinite as the dual of an infinite metrizable precompact group and $\Gamma^{\perp}$ is closed in $G$, we conclude that $G / \Gamma^{\perp}$ is a countably infinite Hausdorff quotient of $G$. This completes the proof.

As an obvious corollary we obtain:

Corollary 2.6 Let $G$ be a precompact abelian group. If $G_{p}^{\wedge}$ does not contain non-trivial convergent sequences, then no infinite Hausdorff continuous homomorphic image of $G$ is countable.

Let us turn to torsion abelian groups. In this case, we characterize the existence of nontrivial convergent sequences in the dual group $G_{p}^{\wedge}$ by any of the equivalent conditions on $G$ given in Proposition 2.5.

In the proof of Theorem 2.7 we denote by $D^{(\omega)}$ the subgroup of $D^{\omega}$ which consists of all elements which differ from the identity of $D$ on at most finitely many coordinates.

Theorem 2.7 For a precompact torsion abelian group $G$, the following conditions are equivalent:

(1) G has a countably infinite Hausdorff quotient;

(2) G has a countably infinite Hausdorff homomorphic image; 
(4) $G_{p}^{\wedge}$ contains a non-trivial convergent sequence.

Proof. The equivalence of (1), (2) and (3) was established in Proposition 2.5. The implication $(3) \Rightarrow(4)$ is evident since an infinite precompact group is non-discrete. Therefore we only need to verify that $(4) \Rightarrow(2)$.

Let $\left\{\chi_{n}: n \in \omega\right\}$ be a non-trivial sequence converging to the identity element of $G_{p}^{\wedge}$. We can assume that $\chi_{n} \neq \chi_{m}$ if $n \neq m$. Let $f$ be the diagonal product of the family $\left\{\chi_{n}: n \in \omega\right\}$. Clearly $f$ is a continuous homomorphism of $G$ to the product group $\mathbb{T}^{\omega}$. Take an arbitrary element $x \in G$ with $x \neq e$ and let $m$ be the order of $x$. Then $m$ is finite since $G$ is a torsion group. Each value $\chi_{n}(x)$ belongs to the cyclic subgroup of $\mathbb{T}$ generated by an element $b \in \mathbb{T}$ of order $m$. Since the sequence $\left\{\chi_{n}(x): n \in \omega\right\}$ converges to $1 \in \mathbb{T}$ for every $x \in G$, the latter implies that there is an integer $n(x) \geq 0$ such that $\chi_{n}(x)=1$ for all $n \geq n(x)$. In other words, $f(x) \in D^{(\omega)}$ for all $x \in G$, where $D$ is the torsion subgroup of $\mathbb{T}$. This implies that the image $f(G)$ is countable.

We claim that $f(G)$ is infinite. Indeed, it follows from our definition of $f$ that for every $n \in \omega$, there exists a continuous character $\mu_{n}$ of $f(G)$ satisfying $\chi_{n}=\mu_{n} \circ f$. Notice that the characters $f^{\wedge}\left(\mu_{n}\right)=\mu_{n} \circ f=\chi_{n}$ of the group $G$ are pairwise distinct, so the set $\left\{\mu_{n}: n \in \omega\right\} \subset f(G)_{p}^{\wedge}$ is infinite. Hence the group $f(G)$ is infinite as well.

\section{Compact subsets of dual groups}

In this section we are concerned with the topological groups that are not necessarily torsion. First, we present a machinery for finding arbitrary compact subsets in the duals of precompact abelian groups. In order to do so, a basic ingredient will be the group $C_{p}(K, \mathbb{T})$ of all continuous functions of a compact space $K$ into the torus $\mathbb{T}$, equipped with the pointwise convergence topology.

Since $C_{p}(K, \mathbb{T})$ is dense in the compact group $\mathbb{T}^{K}$, it follows that the dual group of $C_{p}(K, \mathbb{T})$ is algebraically isomorphic to $A(K)$, the free abelian topological group generated by $K$. Taking into account that, throughout this article, the dual group is always equipped with the pointwise convergence topology, we have that the topological dual of $C_{p}(K, \mathbb{T})$ is the group $A(K)$ 
equipped with the topology of pointwise convergence on elements $C(K, \mathbb{T})$. This topology coincides with the Bohr topology of the free abelian group $A(K)$, that is the topology of pointwise convergence on the elements of $\widehat{A(K)}$ (see [13] and the references therein). Thus we have:

Proposition 3.1 If $K$ is a compact space, then the dual group of $C_{p}(K, \mathbb{T})$ endowed with the pointwise convergence topology is topologically isomorphic to the free abelian topological group $A(K)$ on $K$ equipped with its Bohr topology.

We remark that the free abelian group $A(K)$ respects compactness, which means that every Bohr-compact subset of $A(K)$ is also compact in $A(K)$ (see [13, Corollary 4.20]).

Definition 3.2 Given a compact space $K$, we say that a subgroup $G$ of $C_{p}(K, \mathbb{T})$ is separating if for any distinct elements $x, y \in K$, there is $g \in G$ such that $g(x) \neq g(y)$.

Let $G$ be a topological abelian group. As a consequence of Proposition 3.1 we obtain the following result:

Proposition 3.3 The dual $G_{p}^{\wedge}$ of a precompact abelian group $G$ contains a copy of a compact space $K$ if and only if there is a continuous homomorphism of $G$ onto a separating subgroup of $C_{p}(K, \mathbb{T})$.

Proof. Sufficiency: Let $\varphi: G \longrightarrow H$ be a continuous homomorphism onto a separating subgroup $H \subseteq C_{p}(K, \mathbb{T})$. We have that $A(K)$ is algebraically isomorphic to $C_{p}(K, \mathbb{T})^{\wedge}$. Consider the continuous dual homomorphism

$$
\varphi^{\wedge}:\left(A(K), t_{p}(C(K, \mathbb{T}))\right) \longrightarrow G_{p}^{\wedge}
$$

Since $\varphi(G)$ is separating, it follows that $\varphi^{\wedge} \uparrow K$ is one-to-one.

Necessity: Assume that $K \subseteq G_{p}^{\wedge}$. Then there is a continuous homomorphism $\phi:\left(A(K), t_{p}(C(K, \mathbb{T}))\right) \rightarrow G_{p}^{\wedge}$, which is the identity map on $K$. Therefore, $\phi^{\wedge}(G)$ is a separating subgroup of $C_{p}(K, \mathbb{T})$, where $\phi^{\wedge}: G \rightarrow C_{p}(K, \mathbb{T})$ is the dual homomorphism.

Proposition 3.3 reminds a similar situation which can be encountered in the classical theorem of Peter and Weyl for compact groups (see [19]). 
Corollary 3.4 There are precompact abelian groups whose (precompact) dual groups contain infinite compact subsets but no non-trivial convergent sequences.

Proof. By Proposition 3.1, the dual group of $C_{p}(\beta \omega, \mathbb{T})$ is topologically isomorphic to $A(\beta \omega)$ equipped with the topology of pointwise convergence on $C(\beta \omega, \mathbb{T})$ and the latter group contains a copy of $\beta \omega$. Since $A(\beta \omega)$ does not contain nontrivial convergent sequences (see [10, Theorem 3.4]), neither does $\left(A(\beta \omega), t_{p}(C(\beta \omega, \mathbb{T}))\right.$. This completes the proof.

Remark 3.5 An infinite compact space $K$ is Efimov if $K$ does not contain either nontrivial convergent sequences or $\beta \omega$. Proposition 3.1 implies that the existence of precompact abelian groups whose dual groups contain a compact Efimov subspace is consistent with $Z F C$ (see [11]).

We can now characterize the groups whose dual groups do contain nontrivial convergent sequences. We denote by $c_{0}(\mathbb{T})$ the set of all sequences in $\mathbb{T}$ converging to 1 . Each such a sequence is considered as an element of the compact group $\mathbb{T}^{\mathbb{N}}$. Hence $c_{0}(\mathbb{T})$ can be identified with a subgroup of $\mathbb{T}^{\mathbb{N}}$ which is equipped with the subspace topology.

Theorem 3.6 Let $G$ be a precompact abelian group. Then the dual group $G_{p}^{\wedge}$ contains non-trivial convergent sequences if and only if there is a continuous homomorphism of $G$ onto a separating subgroup of $c_{0}(\mathbb{T})$.

Proof. Let $K:=\{1 / n: n \in \mathbb{N}\} \cup\{0\}$ be a nontrivial convergent sequence. It is easy to see that $C_{p}(K, \mathbb{T})$ is topologically isomorphic to $c_{0}(\mathbb{T})$ equipped with the topology inherited from $\mathbb{T}^{\mathbb{N}}$. Then it suffices to apply Proposition 3.3.

The group $c_{0}(\mathbb{T})$ equipped with the sup-metric is complete and, hence, is a Polish topological group. Therefore, there exist precompact abelian groups admitting a finer non-discrete Polish topological group topology and whose dual groups contain non-trivial convergent sequences.

In the fall of 1990's several specialists in the Pontryagin duality theory were discussing the problem of whether every precompact reflexive abelian group was compact. Later, several counterexamples to this conjecture appeared in [1] and [14], where the authors established the existence of noncompact pseudocompact reflexive abelian groups. Clearly every pseudocompact space has the Baire property. Afterward, a method of constructing 
precompact non-pseudocompact reflexive abelian groups was presented in [3]. All the groups in [3] with this combination of properties had the Baire property. These facts prompt the new conjecture that all precompact reflexive abelian groups have the Baire property. In Theorem 3.9 we show that this conjecture is false by presenting an example of a precompact reflexive abelian group which is of the first category in itself. Our arguments require two auxiliary results (for the first of them, see [18, Proposition 4.4]).

Lemma 3.7 If $G$ is an infinite pseudocompact abelian group, then all compact subsets of the dual group $G_{p}^{\wedge}$ are finite.

Lemma 3.8 Let $H$ be a closed pseudocompact subgroup of a topological abelian group $G$. If the dual group $(G / H)_{p}^{\wedge}$ does not contain infinite compact subsets (nontrivial convergent sequences), then neither does $G_{p}^{\wedge}$.

Proof. Assume that all compact subsets of $(G / H)_{p}^{\wedge}$ are finite. Let $\pi: G \rightarrow$ $G / H$ be the quotient homomorphism and $\pi^{\wedge}:(G / H)_{p}^{\wedge} \rightarrow G_{p}^{\wedge}$ be the dual homomorphism. Let also $r: G_{p}^{\wedge} \rightarrow H_{p}^{\wedge}$ be the restriction mapping, $r(\chi)=$ $\chi\left\lceil H\right.$ for each $\chi \in G_{p}^{\wedge}$. Clearly $r$ is a continuous homomorphism.

Assume for a contradiction that $K$ is an infinite compact subset of $G_{p}^{\wedge}$. Then $r(K)$ is a compact subset of $H_{p}^{\wedge}$. Since $H$ is pseudocompact, $r(K)$ is finite by Lemma 3.7. Hence the compact set $L=K \cap r^{-1}(\mu) \subseteq G_{p}^{\wedge}$ is infinite for some $\mu \in r(K)$. Take an arbitrary element $\chi_{0} \in L$ and put $L^{*}=L \cdot \chi_{0}^{-1}$. Then $L^{*}$ is an infinite compact subset of $G_{p}^{\wedge}$ and the restriction to $H$ of every element $\nu \in L^{*}$ is the identity of $H_{p}^{\wedge}$. Hence for every $\nu \in L^{*}$ there exists a character $\psi \in(G / H)_{p}^{\wedge}$ satisfying $\nu=\psi \circ \pi=\pi^{\wedge}(\psi)$. We conclude, therefore, that $L^{*} \subseteq \pi^{\wedge}\left((G / H)_{p}^{\wedge}\right)$. Since $\pi^{\wedge}$ is a topological monomorphism, the latter inclusion implies that $(G / H)_{p}^{\wedge}$ contains an infinite compact subset. This contradicts the lemma assumptions. We have thus proved that all compact subsets of $G_{p}^{\wedge}$ are finite.

The argument in the case of convergent sequences is almost the same.

A subgroup $H$ of a topological abelian group $G$ is said to be $h$-embedded in $G$ if every homomorphism $h: H \rightarrow \mathbb{T}$ extends to a continuous homomorphism of $G$ to $\mathbb{T}$ (see [1, p. 290]). It is clear from the definition that every homomorphism $h: H \rightarrow \mathbb{T}$ of an $h$-embedded subgroup $H$ of $G$ is continuous.

Let $G$ be a topological abelian group. We recall that the dual group of $G$ endowed with the compact-open topology is denoted by $G^{\wedge}$. If all compact subsets of $G$ are finite, then $G^{\wedge}=G_{p}^{\wedge}$. 
Theorem 3.9 There exists a first category, precompact, reflexive abelian group.

Proof. Since the compact group $\mathbb{Z}(2)^{\omega}$ is second countable, one can apply [3, Theorem 4.2] to find a pseudocompact abelian group $S$ and a closed pseudocompact subgroup $P$ of $S$ such that the quotient group $S / P$ is topologically isomorphic to $\mathbb{Z}(2)^{\omega}$ and all countable subgroups of $S$ are $h$-embedded in $S$. In what follows we identify the groups $S / P$ and $\mathbb{Z}(2)^{\omega}$. Let $G$ be the subgroup of $\mathbb{Z}(2)^{\omega}$ considered in Theorem 2.3. Denote by $\pi$ the quotient homomorphism of $S$ onto $S / P$. Clearly, $H=\pi^{-1}(G)$ is a precompact subgroup of $S, H / P \cong G$, and $\varphi=\pi \uparrow H$ is an open continuous homomorphism of $H$ onto $G$. As $G$ is of the first category, so is $H$. It remains to verify that the group $H$ is reflexive.

First, since $H$ is a subgroup of $S$, all countable subgroups of $H$ are $h$ embedded in $S$. Applying [1, Proposition 2.1] we see that all compact subsets of $H$ are finite. In particular, $H^{\wedge}=H_{p}^{\wedge}$. Also, it follows from Theorem 2.3 that all compact subsets of $(H / P)_{p}^{\wedge} \cong G_{p}^{\wedge}$ are finite. Since $P$ is pseudocompact, all compact subsets of $P_{p}^{\wedge}$ are finite by Lemma 3.7. Therefore, Lemma 3.8 implies that all compact subsets of $H_{p}^{\wedge}$ are finite as well. We conclude, therefore, that

$$
\left(H^{\wedge}\right)^{\wedge}=\left(H_{p}^{\wedge}\right)^{\wedge}=\left(H_{p}^{\wedge}\right)_{p}^{\wedge}
$$

Also, the canonical evaluation mapping of $H$ to $\left(H_{p}^{\wedge}\right)_{p}^{\wedge}$ is a topological isomorphism of $H$ onto $\left(H_{p}^{\wedge}\right)_{p}^{\wedge}$ [24]. Hence the group $H$ is reflexive.

We finish with the following problem mentioned in the introduction:

Problem 3.10 Let $G$ be a precompact topological abelian group with the Baire property. Is it true that all compact subsets of $G_{p}^{\wedge}$ are finite?

Acknowledgements. The authors are grateful to the anonymous referees for careful reading of the original version of the article and helpful comments.

\section{References}

[1] S. Ardanza-Trevijano, M. J. Chasco, X. Domínguez, M. G. Tkachenko, Precompact non-compact reflexive Abelian groups, Forum Math. 24 (2012), no. 2, 289-302. 
[2] A. Arhangel'skii, M. Tkachenko, Topological Groups and Related Structures, Atlantis Studies in Mathematics, vol. I, Atlantis Press, Paris; World Scientific Publishing Co. Pte. Ltd., Hackensack, NJ, 2008.

[3] M. Bruguera, M. Tkachenko, Pontryagin duality in the class of precompact Abelian groups and the Baire property, J. Pure Appl. Algebra 216 (12) (2012), 2636-2647.

[4] M. J. Chasco, X. Domínguez, M. Tkachenko, Duality properties of bounded torsion topological abelian groups, J. Math. Anal. Appl. 448 (2) (2017), 968-981.

[5] W.W. Comfort, K.A. Ross, Topologies induced by groups of character, Fund. Math. 55 (1964), 283-291.

[6] W.W. Comfort, F. J. Trigos-Arrieta, T.S. Wu, The Bohr compactification, modulo a metrizable subgroup, Fund. Math. 143 (1993), 119-136.

[7] D. Dikranjan, S.S. Gabriyelyan, On characterized subgroups of compact abelian groups, Topol. Appl. 160 (2013), 2427-2442.

[8] D. Dikranjan, S. S. Gabriyelyan, V. Tarieladze, Characterizing sequences for precompact group topologies, J. Math. Anal. Appl. 412 (2014), 505-519.

[9] Dikranjan, D. N., I. Prodanov, L. Stoyanov, Topological groups: characters, dualities and minimal group topologies, Monographs and Text in Pure and Applied Mathematics 130, Marcel Dekker Inc., New York-Basel, 1989.

[10] K. Eda, H. Ohta, K. Yamada, Prime subspaces in free topological groups, Topol. Appl. 62 (1995) 163-171.

[11] V.V. Fedorčuk, A compact space having the cardinality of the continuum with no convergent sequences, Math. Proc. Cambridge Philos. Soc. 81 (1977), no. $2,177-181$.

[12] I. Fleischer, T. Traynor, Continuity of homomorphisms on a Baire group, Proc. Amer. Math. Soc. 93 (2) (1985), 367-368.

[13] J. Galindo, S. Hernández, The concept of boundedness and the Bohr compactification of a MAP Abelian group, Fund. Math. 159 (1999), 195-218.

[14] J. Galindo, S. Macario, Pseudocompact group topologies with no infinite compact subsets, J. Pure Appl. Algebra 215 (2011), no. 4, 655-663. 
[15] I. Glicksberg, Uniform boundedness for groups, Canad. J. Math. 14 (1962), 269-276.

[16] P. R. Halmos, Measure Theory, Van Nostrand, New York 1950.

[17] J.E. Hart and K. Kunen, Limits in function spaces and compact groups, Topol. Appl. 151 (2005), 157-168.

[18] S. Hernández and S. Macario, Dual properties in totally bounded Abelian groups, Arch. Math. 80 (2003), 271-283.

[19] E. Hewitt and K. A. Ross, Abstract Harmonic Analysis, Vol. I, Grundlehren Math. Wiss. 115, Springer, Berlin, 1963.

[20] S. Kaplan, Extension of the Pontrjagin duality I: Infinite products, Duke Math. J. 15 (1948), 649-658.

[21] D. J. Lutzer, R. A. McCoy, Category in function spaces, I, Pacific J. Math. 90 (1980), no. 1, 145-168.

[22] S. A. Morris, Pontryagin duality and the structure of locally compact Abelian groups, London Math. Soc. Lecture Notes Series 29 Cambridge Univ. Press 1977.

[23] I. Prodanov, Elementary example of a group without characters, In: Mathematics and Mathematical Education, pp. 79-81 (1981). Proc. Ninth Spring Conf. (April, 1980) of the Bulgarian Math. Soc. at Slunchev brjag (Bulgarian Acad. Sci., Sophia, Bulgaria).

[24] S. U. Raczkowski and F. J. Trigos-Arrieta, Duality of totally bounded Abelian groups, Bol. Soc. Mat. Mex. III, Ser. 7 no. 1 (2001), 1-12.

M.V. Ferrer

Dept. de Matemáticas, Universitat Jaume I, Campus Riu Sec s/n, 12071 Castellón, Spain.

e-mail: mferrer@mat.uji.es

S. Hernández

Dept. de Matemáticas, Universitat Jaume I, Campus Riu Sec s/n, 12071 Castellón, Spain.

e-mail: hernande@mat.uji.es

M. Tkachenko

Dept. de Matemáticas, Universidad Autónoma Metropolitana, Av. San Rafael 
Atlixco \# 186, Col. Vicentina, Iztapalapa, C.P. 09340, Ciudad de México, Mexico. e-mail: mich@xanum.uam.mx 